\title{
Facial Reconstruction: Solving Mysteries and Rewriting Histories
}

\author{
M.K. Sunil ${ }^{1}$, Roopika Handa ${ }^{2}$, Anudeep Raina ${ }^{3}$, Suranagama Lehri ${ }^{4}$, Vasundhara ${ }^{4}$, Aiman Mahfooz ${ }^{4}$ \\ ${ }^{1}$ Professor and Head, ${ }^{2}$ Professor, ${ }^{3}$ Reader, ${ }^{4}$ PG Student, Dept. of Oral medicine and Radiology
}

\begin{abstract}
Facial reconstruction is a multidisciplinary field that holds the ability to identify a missing person from the scratch. It comes into play when all other techniques fail. Over the years, the journey of facial reconstruction has taken many noteworthy turns ands has emerged as a triumph. There have been several attempts from two dimensional techniques which utilize the soft tissue depth to the more recent three dimensional technology in reconstructing the facial features on the skull. From crime cases to the distress caused by natural disasters, facial reconstruction solves it all.
\end{abstract}

Keywords: Facial Reconstruction, Manual Processing, Three dimensional printing.

\section{Introduction}

"The real face is not behind the mask, it is actually with the mask"; and facial reconstruction is the science as well as art that fabricates the veil which beholds the identity of a person. Faces are fascinating with intriguing histories and with the help of forensic advancements, these can be unraveled to crack crimes and help find families their loved ones.

Facial reconstruction is mainly used in two principal branches of science: forensic science and archaeology. Skulls are indestructible for centuries and thus are being utilized for years to teach anatomy lessons and more recently have been identified as a major means to discover a person's identity. ${ }^{2}$

The face of an individual has several different types of exclusive features and thus, is of great importance in identification and recognition of a person. Forensic facial reconstruction is an alternative method in the

\section{Corresponding Author:}

\section{Dr. M.K. Sunil}

Professor and Head, Dept. of Oral medicine and Radiology, Teerthanker Mahaveer Dental College and Research Center, Delhi Road Moradabad UP e-mail: preethiprajana@gmail.com identification process where there is little or no other evidence available. $^{3}$

Hence, in this ellaborative review of literature, we have outlined the history, the process, the manual and advanced methodologies and number of commercially available soft wares for facial reconstruction.

The Historical Outline: The history has witnessed, a multitude of different traditions to deal with the dead. Among the various rituals, the first evidence that the skull was used for remembering the dead dates from the Neolithic Age ${ }^{4}$. Similarly, there are multiple examples from the past depicting how special consideration was given to the head or skull.

Few years later, death masks came into exsistence and in the Italian Renaissance period, death-mask art was most appreciated. Artists from northern Italy were the first to provide wax models for doctors and surgeons.

Nineteenth century marked the beginning of crime detection and in 1895, a German anatomist, Wilheim His did the first facial reconstruction. He reconstructed the face of German composer Johann Sebastein Bach .Another breakthrough came with the advent of "Welcker Facial Reconstruction Technique" by a Welcker, a German physiologist and anatomist. He utilized the average soft tissue depths to construct the mask over the dried skull. ${ }^{1}$ 
In 1946, Wilton Maria Krogmann, brought modifications in the existing method by documenting five basic principles of reconstruction of soft tissues of the face i.e. the relation of eyeball to orbit, the shape of nose tip, the ear location, the mouth width and the ear length ${ }^{1}$

Forensic facial reconstruction took a quatum leap in the 1980s, when Computerized reconstruction was first studied at London College University. Here, at the university, the pioneers carried out a cranial reconstruction procedure using a laser like scanner and video camera.

The Process: The main focus of facial reconstruction is to reinstate the characteristics and features to the details of the dried skull. The creation of the face from the skull is a procedure of approximation., ${ }^{2,5}$

The traditional facial reconstruction method are based on manual procedures, producing $2 \mathrm{D}$ portraits or 3D sculptures.

\section{These method basically consists of three steps:}

- Creating a replica of the raw skull with a sparse set of anatomical landmarks

- apply an average soft tissue thickness to each skull landmark in order to estimate a corresponding landmark on the face

- draw up or sculpt a face fitting the estimated landmarks.

The process of facial reconstruction demands a multidisciplinary approach, where forensic artists, archaeologists, medical team, all team up to reconstruct a perosn's identity. Finally a qualitative comparison is made between facial reconstructions of a real-case skull, based on two typical static face models ${ }^{2,10}$

However, one reconstruction might take several days to months. In order to alleviate these difficulties, several computer graphic software packages have been developed. These advancements utilize the same basic procedure of manual processing, which permits to take advantage of both human expertise and the precision of softwares. The main aim of forensic facial approximations is to promote recognition of a deceased person and all the processes and methodologies are designed to achieve an accurate forensic facial approximation (FFA $)^{5,9}$

\section{The Methodologies}

Methodologies of facial reconstruction involves:

Two Dimensional Reconstruction

3D Manual Reconstruction

Three dimensional printing for facial reconstruction

Two-dimensional reconstruction: In 1980s, Karen Taylor in Austin, Texas developed the construction of face engaging the soft tissue depth estimates. Two dimensional reconstruction focuses on this principle and brings into role the antemortem photographs of an individual.

Moreover, these days certain software programmes like CARESTM or CARES (Computer Assisted Recovery Enhancement System) and FACES (Forensic Anthropology Computer Enhancement System) etc quickly produce $2 \mathrm{D}$ reconstruction which can be edited and manipulated. These softwares work by digitization radiographs, photographs which will replicate an electronically altered version of the image. ${ }^{7}$

3D Manual Reconstruction: This method also needs both an artist and a forensic anthropologist. This method is similar to two dimensional method as it also requires the use of tissue depth markers of specified lengths to represent different soft tissue depths. The markers are inserted into small holes on the skull cast at specific strategic points or landmarks. In the computerized method, computer software produces reconstruction by using scanned and stock photographs. ${ }^{7,8}$

Legends: Here is a diagramatic presentation of the model generation using three dimesional and CT scans and processing them in the software: (diagram 1). 


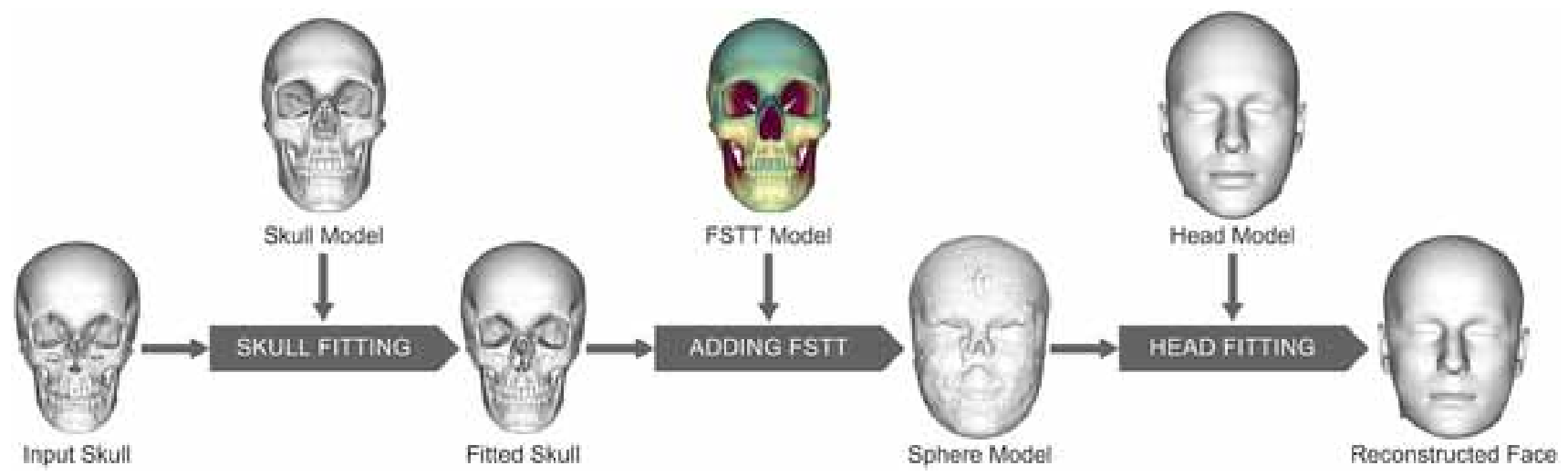

Three dimensional printing for facial whether it is used for forensics or archaeological reconstruction: This method of facial reconstruction purposes. Reconstruction can be processed through is efficient, accurate and noninvasive to the remains, Abode Photoshop for final rendering. ${ }^{6}$

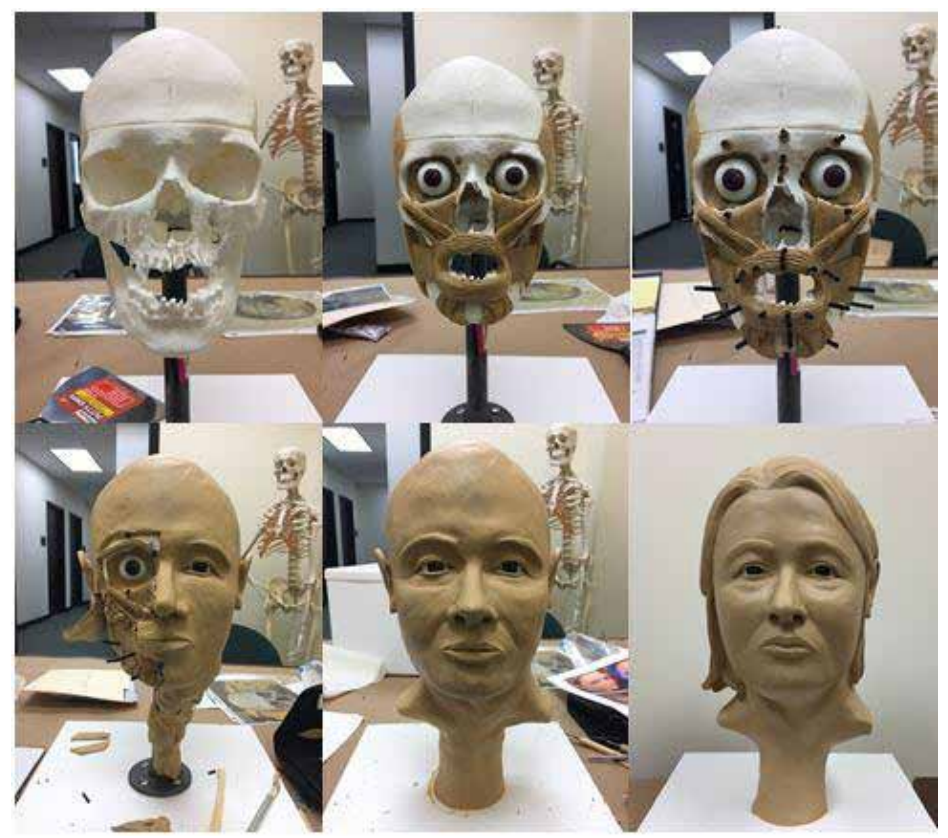

3D Printed Facial Reconstruction (Diagram 2)

\section{Conclusion}

Forensic facial reconstruction provides a non invasive and relatively simple approach to identify a missing person. This is one of the most crucial gateway to solve crime scenes as well as help families find their lost ones. This technique is not only used for identification of individuals from skeletal remains but is also used for archaeological research purposes. Moreover with advent of three dimensional technologies, the whole process has become relatively quick and simpler, opening new gates to this amazing field.
Ethical Clearance: Approved from the etical committee.

\section{Source of Funding: Nil}

Conflict of Interest: None declared

Source of a Support: Nil

\section{References}

1. History of facial reconstruction Laura Verzé, Acta bio-medica: Atenei Parmensis. May 2009. 
2. A method for automatic forensic facial reconstruction based on dense statistics of soft tissue thickness, Thomas Gietzen ID1*, Robert Brylka1, Jascha Achenbach ID2, Katja zum Hebel 3, Elmar Scho "mer4, Mario Botsch 2, Ulrich Schwanecke1, Ralf Schulze5, journal.pone.0210257 January 23, 2019.

3. Turner W, Tu P, Kelliher T, Brown R. Computeraided forensics: facial [15] reconstruction. Stud Health Technol Inform. 2006;119:550-55

4. Fernandes CM, Seera Mda C, da Silva JV, Noritomi PY, Pereira FD, Melani RF. [3] Tests of one Brazilian facial reconstruction method using three soft tissue depth sets and familiar ascessors. Forensic Sci Int. 2012;214:211e1-1e7

5. A facial reconstruction method based on new mesh deformation techniques, Maya de Buhan \& Chiara Nardoni: https://doi.org/10.1080/20961790.2018.1 469185
6. Forensic Facial Reconstruction using Computer Modeling Software,Stephanie L. Davy1, Timothy Gilbert2, Damian Schofield3, Martin P. Evison1

7. Forensic Facial Reconstruction: The Final Frontier, Sonia Gup Ta1, Vinee Ta Gup Ta2, HiTeSH Vij3, Ruc Hieka Vij4, nu Tan TyaGi5

8. Turner WD, Brown RE, Kelliher TP, TuPH, Taister MA, Miller KW.A novel method of automated skull registration for forensic facial approximation. Forensic Sci Int 2005

9. Stephan CN. Do resemblance ratings measure the accuracy of facial approximations? J Forensic Sci 2002

10. Claes $\mathrm{P}$, Vandermeulen $\mathrm{D}$, De Greef $\mathrm{S}$, et al. Craniofacial reconstruction using a combined statistical model of face shape and soft tissue depths: methodology and validation. Forensic Sci Int. 2006 